\title{
Phylogeographical autocorrelation of phenotypic evolution in honey bees (Apis mellifera L.)
}

\author{
JOSÉ ALEXANDRE FELIZOLA DINIZ-FILHO*†, STEFAN FUCHS: \\ \& MARIA CRISTINA ARIAS§ \\ $\nmid$ Departamento de Biologia Geral, Instituto de Ciências Biológicas, Universidade Federal de Goiás, C.P. 131, \\ 74.001-970, Goiânia, GO, Brasil, §Institut für Bienenkunde (Polytechnische Gesellschaft), Fachbereich Biologie \\ der J. W. Goethe-Universität Frankfurt am Main, Karl-von-Frisch-Weg 2, D-61440 Oberursel, Germany and \\ $\S$ Departamento de Biologia, Instituto de Biociências, Universidade de São Paulo, C.P. 11461, 05422-970, \\ São Paulo, SP, Brasil
}

The analysis of phenotypic divergence among local populations within a species has been traditionally performed in a spatial context, although advances in genetic analysis using mtDNA have permitted a simultaneous evaluation of geographical and historical patterns of variation, so-called phylogeographical analysis. In this paper, we combine these two dimensions of variation (geographical space and phylogenetic history) to evaluate patterns of phenotypic evolution in honey bees (Apis mellifera L.). Data on 39 phenotypic traits, derived from 417 colonies grouped into 14 subspecies, were analysed using autocorrelation methods. Mantel tests indicated that the relationship between phenotypic divergence, estimated by Euclidean distances among subspecies' morphological centroids, was significant both when compared to geographical distance $(r=0.371 ; P<0.01)$ and to genetic distance (estimated as sequence divergence (\%) in a mtDNA region encompassing part of the NADH dehydrogenase subunit 2 and isoleucine transfer RNA $(r=0.329 ; P<0.01))$. For the analysis of each trait, the effects of the geographical co-ordinates (latitude and longitude of subspecies geographical range) and of the phylogenetic patterns (defined by eigenvectors of the genetic distance matrix) on phenotypic variation were simultaneously analysed using an extension of a recently developed model, called Phylogenetic Eigenvector Regression (PVR). In general terms, the partial regression slopes indicated that the variation in the characters traditionally associated with adaptive processes, such as body and wing size, were better explained by geographical position. However, characters usually thought to be neutral, such as wing venation angle, were more associated with phylogeny. This is expected because PVR can be interpreted as a partition model, in which adaptive variation tends to be independent of phylogeny (and, in this case, associated with geography). In addition, the first principal component derived from the expected values of the model for each trait, which can be interpreted as the phenotypic variation predicted by phylogeny, is more structured in a north-south cline than are the original data, supporting an adaptive interpretation. The phylogeographical autocorrelation analyses performed in this study show that different traits are more related to one of the two dimensions of variation (geography and phylogeny), and these patterns can furnish insights into the nature of phenotypic evolution in these organisms.

Keywords: honey bees, multivariate morphometrics, phenotypic evolution, phylogenetic autocorrelation, phylogeography, spatial autocorrelation.

\section{Introduction}

The analysis of population structure and phenotypic divergence among local populations within a species has

*Correspondence. E-mail: diniz@icbl.ufg.br

(C) 1999 The Genetical Society of Great Britain. been traditionally performed in a spatial context (Sokal, 1986; Roderick, 1996). More recently, researchers have started to use mitochondrial DNA (mtDNA) variation to track sequential patterns of population differentiation, superimposing these historical links on geographical space in order to make inferences about microevolutionary processes at the populational level. 
These inferences include the relative roles of the restriction of gene flow by geographical and historical barriers, the stochastic colonization success of some lineages, and the effects of population bottlenecks (Zink, 1996). This approach has been called intraspecific phylogeography (Avise et al., 1987; Crozier, 1990; Roderick, 1996). It can be also extended to the analysis of multiple species in the same geographical area, searching for common causes of their population structures (Zink, 1996). A neutral diffusion model of populations that continuously spread through geographical space, similar to an isolation-by-distance model in population genetics, predicts a monotonic relationship between the phylogenetic and geographical distances between (pairs of) local populations. However, although phylogenetic and geographical distances are usually correlated, even within populations, it is important that these patterns of variation among populations are estimated independently, such as in phylogeographical analyses, in order to make inferences about processes of microevolutionary differentiation in other traits (Roderick, 1996).

Knowledge of these two referential dimensions of phenotypic variability (phylogeny and geographical space) is also important because recent work in ecology and evolutionary biology has demonstrated that taxa (both species and subspecies) do not provide independent observations for statistical analyses of correlated evolution. This occurs because these traits are usually autocorrelated across historical and geographical dimensions. Populations share ancestors at distinct points of time (there is a phylogenetic connection among them) (Felsenstein, 1985; Harvey \& Pagel, 1991) and, simultaneously, some processes influencing population differentiation, especially migration and dispersal, are stronger between populations closer together in geographical space (Sokal \& Oden, 1978a,b; Sokal \& Jacquez, 1991). These autocorrelation patterns in phenotypes, across both phylogenetic and spatial dimensions, increase the Type I error and disturb parameter estimation when inferring adaptations through standard correlation or regression analyses between characters, or between characters and other components of environmental variation (Martins \& Hansen, 1996). However, it was also simultaneously recognized that patterns of historical and spatial autocorrelation could be used to improve knowledge about evolutionary processes acting in phenotypic divergence (Sokal \& Jacquez, 1991; Hansen \& Martins, 1996). Working at the population level permits the simultaneous evaluation of these two effects.

Once phylogeographical patterns have been established, it is interesting to map the character variation over these patterns, in order to make specific inferences about the evolutionary processes acting on phenotypic traits. Variation in a neutral character, for example, should track the phylogenetic structure by a purely stochastic process (Brownian motion producing phylogenetic inertia) and will possess only a partial (in a statistical sense) relationship with geographical space. On the other hand, adaptations to local or regional environmental variation can be independent of phylogenetic inertia, being structured on geographical space if the adaptive range is larger than interpopulation spatial distances (Sokal \& Jacquez, 1991). Edwards \& Kot (1995) recently applied autocorrelation methods to variation within species, but analysed only the relationship between morphology and historical links between individuals (established using mtDNA), independently of their geographical locations.

The honey bee (Apis mellifera L.) shows patterns of biological variation rarely found in other animal groups, because this species has an unusually large geographical distribution, which encompasses very distinct environmental conditions (Ruttner, 1988). Many local types and subspecies have been recognized, and the evolution of population structure at distinct geographical scales has been attributed both to adaptive and to stochastic processes (Wagner, 1990). Ecogeographical rules, including Bergmann's and Allen's rules, have been widely used to explain morphological variation and adaptation (Ruttner, 1988), notwithstanding the recent discussion about the microevolutionary processes involved in the origin of these patterns (Cushman et al., 1993; Van Voorhiers, 1996). In recent years, the analysis of population differentiation at the molecular level, especially of allozymes and mtDNA, has aided our understanding of the detailed processes of population differentiation among honey bee populations, by contrasting morphological, behavioural, geographical and molecular variation (Cornuet \& Garnery, 1991; Arias \& Sheppard, 1996).

Arias \& Sheppard (1996) recently used a mtDNA region encompassing part of the $N A D H$ dehydrogenase subunit 2 gene and of the tRNA ILE to reconstruct the phylogenetic relationships among 14 geographical races of A. mellifera, and discussed the correspondence between morphological and molecular classifications. The phylogenies obtained using mtDNA can be understood as null models of relationship among subspecies, because they were estimated independently of their geographical position and morphological variation. The objective of this paper is to evaluate the correspondence between historical and geographical patterns of differentiation among subspecies of $A$. mellifera and to establish in what ways the phenotypic variation is related to each pattern. This can provide insights about the evolutionary processes acting on different traits of honey bees worldwide, identifying traits as being associated with adaptive or with stochastic processes of population differentiation. 


\section{Materials and methods}

\section{Data set}

For this study, we analysed the 14 subspecies of A. mellifera that were previously studied by Arias \& Sheppard (1996) (Table 1). Morphological data for these subspecies were obtained from the Oberursel morphometric data bank (Germany), in which samples have been collected and analysed since the early 1960s, mainly by F. Ruttner and his collaborators (see Ruttner, 1988; for details). Because morphometric data and phylogenies were not exactly derived from the same locations, we used a conservative approach in combining morphological, molecular and geographical data of the subspecies. Because the bee colonies used for constructing phylogenies were classified as typical samples of the races studied by morphometric analyses (see below), we selected from the Oberursel data bank only the samples (colonies) that possessed a probability of $99 \%$ of belonging to a given subspecies, using previously established procedures of discriminant analyses (Ruttner, 1988).

For the 14 subspecies, the average values for 39 morphological traits of worker bees were derived from a total of 417 colonies, with approximately 20 bees per colony. Sample sizes per subspecies vary (Table 1). These traits have been traditionally used for morphological analysis of honey bee populations worldwide

Table 1 Subspecies of Apis mellifera analysed in this study and their respective sample sizes ( $n$, number of colonies, with approximately 20 bees per colony), geographical coordinates of the centre of their geographical ranges and the two eigenvectors (PHY1 and PHY2) derived from the double-centred phylogenetic distance matrix $\mathbf{D}$

\begin{tabular}{lrrrrrr}
\hline & & & & \multicolumn{2}{c}{ Eigenvectors } \\
\cline { 5 - 6 } Subspecies & $n$ & \multicolumn{2}{c}{ Latitude } & Longitude & PHY1 & PHY2 \\
\hline A.m. mellifera & 71 & $52^{\circ} \mathrm{N}$ & $5^{\circ} \mathrm{E}$ & 0.077 & 0.105 \\
A. m. carnica & 57 & $47^{\circ} \mathrm{N}$ & $15^{\circ} \mathrm{E}$ & 0.085 & -0.018 \\
A. m. ligustica & 33 & $43^{\circ} \mathrm{N}$ & $13^{\circ} \mathrm{E}$ & 0.075 & -0.027 \\
A. m. macedonica & 7 & $42^{\circ} \mathrm{N}$ & $22^{\circ} \mathrm{E}$ & 0.073 & -0.010 \\
A. m. meda & 66 & $38^{\circ} \mathrm{N}$ & $38^{\circ} \mathrm{E}$ & 0.074 & -0.040 \\
A. m. adansonii & 14 & $5^{\circ} \mathrm{N}$ & $15^{\circ} \mathrm{E}$ & -0.027 & -0.025 \\
A. m. capensis & 6 & $33^{\circ} \mathrm{S}$ & $18^{\circ} \mathrm{E}$ & -0.032 & -0.032 \\
A. m. iberica & 53 & $38^{\circ} \mathrm{N}$ & $5^{\circ} \mathrm{W}$ & -0.046 & 0.019 \\
A.m. intermissa & 23 & $35^{\circ} \mathrm{N}$ & $1^{\circ} \mathrm{W}$ & -0.056 & 0.015 \\
A. m. lamarckii & 24 & $27^{\circ} \mathrm{N}$ & $31^{\circ} \mathrm{E}$ & -0.047 & 0.042 \\
A. m. monticola & 30 & $0^{\circ} \mathrm{N}$ & $38^{\circ} \mathrm{E}$ & -0.050 & -0.038 \\
A. m. sahariensis & 7 & $31^{\circ} \mathrm{N}$ & $7^{\circ} \mathrm{W}$ & -0.046 & 0.019 \\
A. m. scutellata & 5 & $10^{\circ} \mathrm{S}$ & $35^{\circ} \mathrm{E}$ & -0.032 & -0.031 \\
A. m. sicula & 21 & $37^{\circ} \mathrm{N}$ & $14^{\circ} \mathrm{E}$ & -0.046 & 0.019 \\
\hline
\end{tabular}

(Ruttner, 1988; Wagner, 1990), and include linear measurements of different body parts and locomotor appendages (legs, buccal apparatus, thorax, tergites and sternites, wings), venation angles of forewing and colour of different parts (labrum, tergites) (Table 2).

\section{Phylogenetic and geographical distances between subspecies}

Initially, matrices of geographical and phylogenetic distances between the 14 subspecies were constructed. For phylogenetic distances, the matrix was obtained directly from the work of Arias \& Sheppard (1996), and estimated as sequence divergence $(\%)$ in a mtDNA region of $688 \mathrm{bp}$, encompassing part of the $N A D H$ dehydrogenase subunit 2 and isoleucine transfer RNA. Because their analysis was carried out by colony, we combined distances between colonies from the same subspecies, and constructed a new phylogenetic distance matrix between pairs of subspecies. In this process, colonies showing disagreement between morphology and mtDNA sequence were excluded, as occurred with $A . m$. ligustica and $A . m$. meda (samples called ITMEL and MEDA1, respectively, in Arias \& Sheppard, 1996). As a result, we included only typical colonies of each race, both in terms of morphology and mtDNA patterns. Although this procedure is artificial, in that it avoided the natural continuous variation among subspecies within species, it was necessary in order to combine geographical, phylogenetic and morphological patterns from different sources. Arias \& Sheppard (1996) constructed trees based on this distance matrix using the neighbour-joining method (NJ) and parsimony, and the trees derived using the same methods from the new matrix produced for this study were essentially the same as the previous trees (Fig. 1). It is also important to note that differences in tree topology also appeared in the original work by Arias \& Sheppard (1996) when using different methods, indicating sensitivity of the pairwise distances to the clustering algorithm. These slight variations do not affect the present study, because all analyses were based directly on phylogenetic distances and not on trees, as is required by Mantel tests and by the PVR method (see below).

Geographical distances between pairs of subspecies were defined as great circle distances, in $\mathrm{km}$, between the centres of their geographical distributions (the centroid of each subspecies distribution; Taylor \& Gotelli, 1994), established using data from Ruttner (1988) (Fig. 1; Table 1). These estimates are always approximate because of difficulties in obtaining a precise location of the distribution based only on collection sites, and because of the natural range dynamics of taxa (Taylor \& 
Table 2 Patterns of phylogeographical variation in morphological traits of Apis mellifera, using multiple regression of traits on phylogenetic (PHY1 and PHY2) and geographical (Latitude and Longitude) vectors simultaneously. Results include the coefficient of determination of the model $\left(R^{2}\right)$, the $F$-value of the ANOva from regression and the standardized partial slope of each vector

\begin{tabular}{|c|c|c|c|c|c|c|c|}
\hline \multirow[b]{2}{*}{ Character } & \multirow[b]{2}{*}{ Acronym } & \multirow[b]{2}{*}{$R^{2}$} & \multirow[b]{2}{*}{$F$} & \multicolumn{4}{|c|}{ Partial slopes } \\
\hline & & & & PHY1 & PHY2 & Latitude & Longitude \\
\hline Hair length & HAIR & 0.767 & $7.407 * *$ & 0.142 & 0.186 & 0.704 & 0.028 \\
\hline Tomentum length & TOMENT & 0.695 & $5.121^{*}$ & 0.344 & -0.028 & 0.590 & 0.416 \\
\hline Tomentum width & TODARK & 0.406 & 1.537 & 0.105 & 0.215 & -0.792 & -0.362 \\
\hline Proboscis length & PROBOSCI & 0.808 & $9.491 * *$ & 0.046 & -0.540 & 0.887 & -0.346 \\
\hline Hind femur length & FEMUR & 0.618 & $3.640^{*}$ & 0.438 & -0.051 & 0.033 & -0.713 \\
\hline Hind tibia length & TIBIA & 0.605 & $3.439 \dagger$ & 0.388 & -0.031 & 0.078 & -0.683 \\
\hline Hind metatarsus length & METLEN & 0.715 & $5.644 *$ & 0.402 & 0.245 & 0.115 & -0.530 \\
\hline Hind metatarsus width & METWID & 0.510 & 2.342 & 0.438 & -0.025 & -0.013 & -0.626 \\
\hline Pigmentation level on tergite 2 & PIGTER2 & 0.109 & 0.275 & -0.001 & -0.118 & -0.068 & 0.213 \\
\hline Pigmentation level on tergite 3 & PIGTER3 & 0.079 & 0.194 & 0.092 & -0.136 & -0.180 & 0.038 \\
\hline Pigmentation level on tergite 4 & PIGTER4 & 0.208 & 0.590 & 0.127 & -0.134 & -0.424 & -0.014 \\
\hline Length of tergite 3 & TERG3 & 0.663 & $4.434 *$ & 0.310 & 0.150 & 0.294 & -0.435 \\
\hline Length of tergite 4 & TERG4 & 0.718 & $5.740^{*}$ & 0.342 & 0.150 & 0.320 & -0.427 \\
\hline Length of sternum 3 & STERN3 & 0.642 & $4.031 *$ & 0.512 & -0.036 & 0.005 & -0.701 \\
\hline Length of wax mirror & WAXLEN & 0.703 & $5.332 *$ & 0.365 & 0.065 & 0.383 & -0.382 \\
\hline Width of wax mirror & WAXWID & 0.767 & $7.401 * *$ & 0.371 & -0.100 & 0.394 & -0.540 \\
\hline Distance between mirrors & WAXDIS & 0.672 & $4.614 *$ & 0.078 & -0.035 & -0.800 & 0.083 \\
\hline Length of sternum 6 & STERN6 & 0.528 & 2.515 & 0.547 & -0.021 & -0.115 & -0.668 \\
\hline Width of sternum 6 & STERNW6 & 0.676 & $4.687^{*}$ & 0.388 & 0.074 & 0.165 & -0.593 \\
\hline Fore wing length & FWINGL & 0.636 & $3.929 *$ & 0.644 & -0.132 & -0.068 & -0.693 \\
\hline Fore wing width & FWINGW & 0.633 & $3.889^{*}$ & 0.737 & -0.385 & -0.082 & -0.586 \\
\hline Pigmentation level of scutellum 1 & PIGSCUT1 & 0.075 & 0.182 & -0.141 & -0.142 & 0.067 & 0.198 \\
\hline Pigmentation level of scutellum 2 & PIGSCUT2 & 0.132 & 0.342 & -0.132 & 0.351 & -0.223 & 0.079 \\
\hline Pigmentation level on labium 1 & PIGLAB1 & 0.014 & 0.033 & -0.093 & -0.116 & 0.159 & -0.019 \\
\hline Pigmentation level on labium 2 & PIGLAB2 & 0.125 & 0.320 & 0.316 & -0.014 & 0.065 & -0.036 \\
\hline Length of cubital vein a & CUB1 & 0.537 & 2.606 & 0.374 & -0.612 & 0.381 & -0.143 \\
\hline Length of cubital vein $b$ & CUB2 & 0.324 & 1.067 & 0.322 & 0.553 & -0.355 & -0.273 \\
\hline Wing venation angle & A4 & 0.708 & $5.449 *$ & -0.356 & 0.645 & -0.575 & 0.263 \\
\hline Wing venation angle & B4 & 0.685 & $4.896^{*}$ & 0.395 & -0.597 & 0.536 & -0.221 \\
\hline Wing venation angle & D7 & 0.490 & 2.164 & 0.287 & 0.631 & -0.895 & 0.277 \\
\hline Wing venation angle & E9 & 0.643 & $4.059^{*}$ & 0.320 & -0.681 & 0.519 & -0.038 \\
\hline Wing venation angle & G18 & 0.546 & $2.708 \dagger$ & -0.443 & 0.355 & -0.143 & -0.289 \\
\hline Wing venation angle & $\mathrm{J} 10$ & 0.541 & 2.652 & 0.403 & -0.479 & -0.149 & 0.140 \\
\hline Wing venation angle & J16 & 0.745 & $6.586^{* *}$ & -0.196 & 0.321 & 0.330 & -0.431 \\
\hline Wing venation angle & K19 & 0.165 & 0.444 & 0.215 & 0.492 & -0.402 & -0.017 \\
\hline Wing venation angle & L13 & 0.321 & 1.063 & -0.451 & 0.263 & 0.228 & -0.070 \\
\hline Wing venation angle & $\mathrm{N} 23$ & 0.597 & $3.334 \dagger$ & 0.270 & 0.237 & 0.406 & -0.147 \\
\hline Wing venation angle & $\mathrm{AO} 26$ & 0.562 & $2.885 \dagger$ & -0.609 & 0.377 & 0.119 & -0.091 \\
\hline Number of hamuli on hind wing & HOOKS & 0.350 & 1.212 & -0.420 & -0.551 & 0.553 & 0.324 \\
\hline
\end{tabular}

$\dagger P<0.10 ; * P<0.05 ; * * P<0.01$.

Gotelli, 1994). Also, the subspecies boundaries are even more imprecise than species boundaries because of local hybridization among adjacent taxa. For subspecies with very large distributions, such as $A$. $m$. mellifera, the locations used to establish the centre of geographical distributions were based on the samples used by Arias \& Sheppard (1996) and Ruttner (1988), in order to maximize the correspondence between morphological and phylogenetic characterization of subspecies.

\section{Data analysis}

The first step in our analysis was to evaluate how the overall morphological patterns were related to 
Fig. 1 Phylogeographical relationships among the 14 subspecies of Apis mellifera analysed in this study. The centres of geographical distribution, used to estimate geographical distances, are given in Table 1, and the phylogeny is given as a neighbour-joining tree based on genetic distances estimated using mtDNA, derived from Arias \& Sheppard (1996).

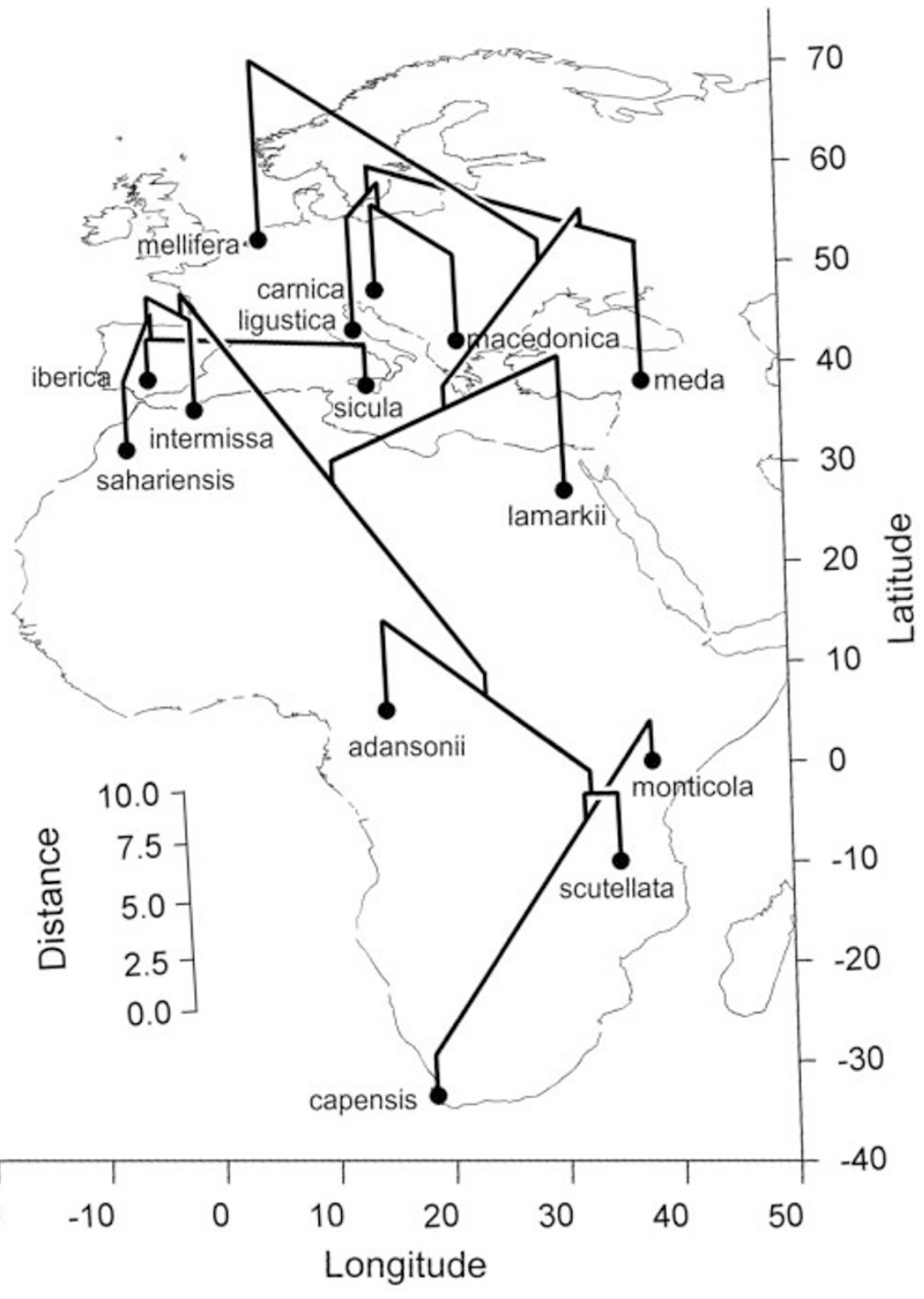

phylogenetic and geographical relationships between subspecies, using Mantel tests (Smouse et al., 1986; Oden \& Sokal, 1992). A matrix with Euclidean distances between (pairs of) subspecies, estimated using the 39 morphological traits (after data standardization because of differences in data type and scale), was compared with the matrices containing phylogenetic or geographical distances between them, previously defined. The Mantel $Z$ statistic is given by

$Z=\sum_{i} \sum_{j}\left(M_{i j} D_{i j}\right)$

where $M_{i j}$ is the element of the pairwise morphological distance matrix $\mathbf{M}$ between subspecies, expressing distance between subspecies $i$ and $j$, and $D_{i j}$ is the element of the matrix $\mathbf{D}$, containing geographical or phylogenetic distances between them. The significance of $Z$ was determined by comparing the observed value with a null distribution constructed by randomization of one of the matrices and recalculation of the statistics many times. For this work, 10000 random $Z$-values were used to define the null distribution of the statistics, using the routine MXCOMP from NTSYS-PC, version 1.5 (Rohlf, 1989). Also, as the $Z$-value is largely dependent upon the magnitude of elements and sample size in the matrices, it was standardized to obtain a matrix correlation coefficient analogous to Pearson productmoment correlation, ranging from -1.0 to 1.0 . Partial Mantel tests (Smouse et al., 1986; Oden \& Sokal, 1992) were also applied to test if correlation between morphology and one predictor persisted when the other predictor was held constant.

Geographical and phylogenetic effects were also analysed for each trait independently. For geographical analyses, a trend surface (Wartenberg, 1985) was used. This technique consists simply of performing a multiple

(C) The Genetical Society of Great Britain, Heredity, 83, 671-680. 
regression on each variable, using geographical co-ordinates (latitude and longitude, and possibly their polynomial expansions) as predictors. In this case, the predictors were the latitude and longitude of the centre of the geographical range of each subspecies.

For evaluating phylogenetic effects, many distinct methods are available today (Harvey \& Pagel, 1991; Martins \& Hansen, 1996). We used in this paper a new approach recently proposed by Diniz-Filho et al. (1998), called the Phylogenetic Eigenvector Regression (PVR) procedure. This method was chosen because of its computational simplicity and facility in performing statistical inferences about the null hypothesis of no phylogenetic effects in data. Also, and more importantly, the results are directly comparable to those obtained with the trend surface analyses previously discussed. The purpose of PVR is to partition the total phenotypic variance $(\mathrm{T})$ into phylogenetic $(\mathrm{P})$ and specific (S) components, in an analogous way to the phylogenetic autoregressive model (Cheverud et al., 1985; Gittleman $\&$ Kot, 1990). This is achieved by regressing each trait on the eigenvectors derived from a Principal Co-ordinate Analysis (Johnson \& Wichern, 1992) applied directly to the phylogenetic distance matrix $\mathbf{D}$. The eigenvectors thus express the variation between the subspecies in the reduced multivariate space of the genetic distances. Formally, the model of PVR is

$\mathbf{Y}=\mathbf{X} \beta+\varepsilon$,

where $\mathbf{Y}$ is the vector of the trait analysed in the subspecies, $\mathbf{X}$ is the matrix of eigenvectors derived from $\mathbf{D}, \beta$ is the vector of regression parameters and $\varepsilon$ is the residual vector. The coefficient of determination of this regression model $\left(R^{2}\right)$ estimates the part of variation in the trait that can be attributed to phylogenetic effects $(\mathbf{X})$, and the test of its statistical significance can be performed with a standard $F$-test (Sokal \& Rohlf, 1995; Diniz-Filho et al., 1998). The residuals of this multiple regression $(\varepsilon)$ express the part of variation in the trait that cannot be explained by phylogenetic distances among taxa, and thus indicate variation in each subspecies independently of the variation in other ones, in a phylogenetic sense. So, they are similar to the specific component $\mathrm{S}$ of the phylogenetic autoregressive model. The number of eigenvectors to be used in the analysis can be defined using many available criteria, but previous comparative work recognized that the broken-stick method is very appropriate (Jackson, 1993; Diniz-Filho et al., 1998).

A multivariate expression of $\mathrm{T}, \mathrm{P}$ and $\mathrm{S}$ components was also obtained, using the scores of a Principal Component Analysis (PCA) (Johnson \& Wichern, 1992) based on the residuals of PVR applied to each trait. Both for the trend surface and PVR, a direct multivariate analysis of effects, extending multiple regressions to a unique Canonical Correlation Analysis (Wartenberg, 1985; Diniz-Filho et al., 1998), was not possible in this study because of the relatively low number of observations in relation to the variables (14 subspecies with 39 traits).

The two multiple regression analyses (trend surface and PVR) were applied to all 39 morphological traits. However, because many characters were related both to geographical and phylogenetic effects, a combined general analysis was also performed, by regressing the traits on the four predictors simultaneously (latitude, longitude and the two eigenvectors of PVR), using standardized partial slopes (Sokal \& Rohlf, 1995) to evaluate their relative magnitudes.

\section{Results}

Mantel tests indicate a significant correlation between phenotypic variation, estimated by Euclidean distances using all morphological characters, and phylogenetic $(r=0.329 ; \quad P=0.008)$ and geographical $(r=0.371$; $P=0.0032)$ distances. Partial Mantel tests were also significant, because correlation between phylogenetic and geographical distances was not significant $(r=0.151 ; P=0.162)$. The correlation between phenotypic and geographical distances, holding phylogeny constant, was equal to $0.332(P=0.009)$, and the correlation between phenotypic and phylogenetic distances, holding geography constant, was equal to 0.304 $(P=0.012)$.

For the analysis of the correlation of each character with both dimensions, multiple regressions were used. For geographical analysis, only latitude and longitude were used as predictors in trend surface, as quadratic expansions did not significantly increase the coefficients of determination of most traits. For phylogenetic analysis, the matrix $\mathbf{D}$, containing genetic distances (\% sequence divergence) based on mtDNA, was doublecentred and submitted to eigenanalysis. Out of 14 eigenvalues extracted from $\mathbf{D}$, two were considered significant under a broken-stick distribution, and explained $82.54 \%$ of the variability. Associated eigenvectors were then retained (Table 1) and the matrix correlation between the original genetic distances and the distances in the bivariate space formed by these first two eigenvectors was equal to 0.944 . This indicates that these two axes contain most of the information in the original genetic distance matrix. So, to evaluate the phylogenetic patterns in each of the 39 morphological characters, they were regressed on these two eigenvectors. 


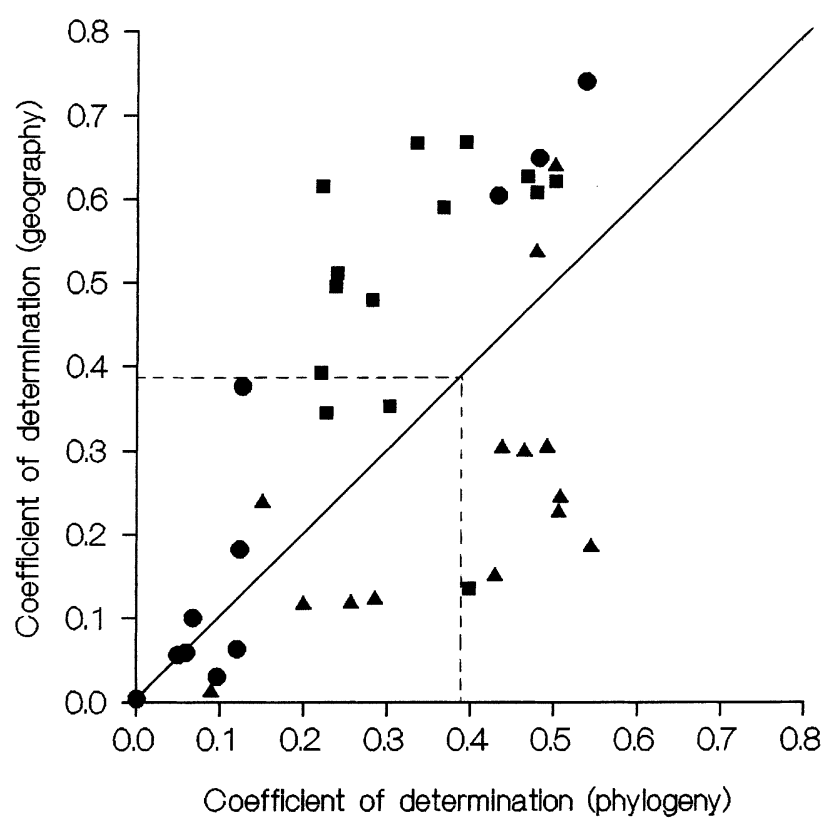

Fig. 2 Comparison of coefficients of determination $\left(R^{2}\right)$ of the two methods (trend surface and PVR) applied to evaluate, respectively, geographical and phylogenetic patterns of the 39 morphological traits of Apis mellifera. The dashed line indicates the critical level of $R^{2}$ and the solid line indicates the $45^{\circ}$ line, i.e. the equal importance of phylogenetic and geographical patterns on phenotypic variation. Triangles represent wing venation characters (angles), squares body size characters and circles colour characters.

The $R^{2}$ values of multiple regression were usually high for both geography and phylogeny, for most characters, supporting the overall test of phenotypic variation using Mantel tests (Fig. 2). It is possible to note, however, that some characters (especially colour traits) do not show significant correlation with any of the dimensions (the critical $R^{2}$ is 0.39 . with 2 and 11 d.f. for the phylogenetic and geographical regression analyses, respectively). On the other hand, some characters are more associated with one of the dimensions, although significantly correlated with both. In graphical terms, they are below or above the line with $45^{\circ}$ that relates the two vectors of $R^{2}$ (Fig. 2). Some characters of wing venation angles (G18, A4, B4, J10, E9, CUB1, AO26) are more related to phylogeny, and some characters based on size measurements (especially PROBOSCI, WAXDIS, WAXWID, WAXLEN, STERNW6, TIBIA and FEMUR) are more related to geography.

Because this last group of characters possess significant correlations with both phylogenetic and geographical dimensions, it is important to consider the two dimensions simultaneously. So, a new multiple regression was applied, using all four vectors as predictors of each of the 39 characters. The standard- ized partial slopes (Table 2) confirmed the results of the previous analyses using the dimensions independently. As previously found, the characters of wing venation tend to be more related to phylogeny, and characters of size measurements tend to be more related to geography.

The PVR allowed the partition of the variation of each trait into phylogenetic and specific components. Thus, it is also possible to use a multivariate technique (PCA), applied to T, P and S values estimated by PVR for each of the 39 characters. The PCA was used to produce a simple multivariate solution to predict which part of the phenotypic variation is expected by the phylogeny and which part is independent of it. Principal component scores were used to evaluate spatial structure on phylogenetic and subspecific patterns of morphological variation.

For the total values ( $\mathrm{T}$, original traits), seven components were extracted, and the first principal component, usually interpreted as an estimate of general body size, explains around $44.37 \%$ of the morphometric variation. This multivariate expression of body size is also spatially structured in a north-south cline (Fig. 3), and the $R^{2}$ from a trend surface analysis applied to the scores was equal to 0.526 . For the $\mathrm{P}$ component, the first eigenvalue explains around $89.12 \%$ of the variability, being the only one significant according to the broken-stick distribution. The map of the first principal component obtained from the $\mathrm{P}$ components indicates a strong north-south cline, with $R^{2}=0.511(P=0.020)$ in a trend surface model (Fig. 4). On the other hand, for the $S$ components, the first principal component explains only $43.34 \%$ of the variability, and there are eight axes with significant eigenvalues. This indicates, as expected, a stronger independence among $\mathrm{S}$ components of the traits. Thus, different patterns of relationship with geographical and phylogenetic distance matrices can be attributed to a stronger specific component. None of the seven axes derived from $\mathrm{S}$ components is spatially structured, indicating that each subspecies really evolved independently after controlling both spatial and phylogenetic effects.

\section{Discussion}

Phylogeographical autocorrelation analyses performed in this study show that morphological variation among subspecies of honey bees can be explained by both geographical and phylogenetic effects. However, different traits are more related to one of the two dimensions of variation, and these patterns can furnish insights about the nature of phenotypic variation.

The three main groups of characters analysed (body size, wing venation angles and colours) behaved 


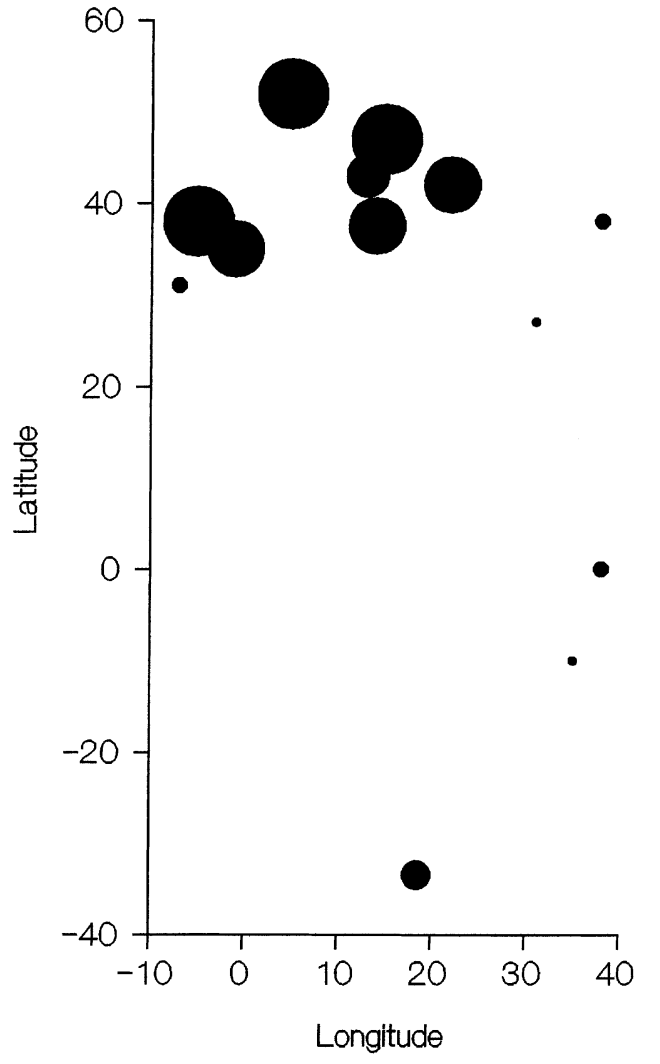

Fig. 3 Schematic map of multivariate morphological variation in the 14 subspecies of Apis mellifera analysed, using the first principal component of the total values of the 39 characters. The size of the circles indicates the relative values of multivariate scores, and larger circles represent higher scores (larger bees).

differently in relation to geography and phylogeny. In general terms, body size is related both to geographical and to phylogenetic patterns (but more with geography), wing venation is related more to phylogeny, and colour is not correlated with either of these two dimensions of variation.

The patterns described above have been usually recognized when analysing global distributions of honey bee phenotypes (Ruttner, 1988; Wagner, 1990). The new and more important information, however, is that the body size variables are much less correlated with phylogenetic effects than with geographical ones, such that the traditional adaptive interpretation is not disturbed by phylogenetic inertial effects (Cheverud et al., 1985; Gittleman \& Kot, 1990). More importantly, the first multivariate axis derived from $\mathrm{P}$ components is strongly structured in geographical space. This indicates that expected phenotypic variation under a mtDNA phylogenetic hypothesis is congruent with a continuous geographical pattern, suggesting in fact that the null model of phylogeographical divergence is supported for morphological variation.

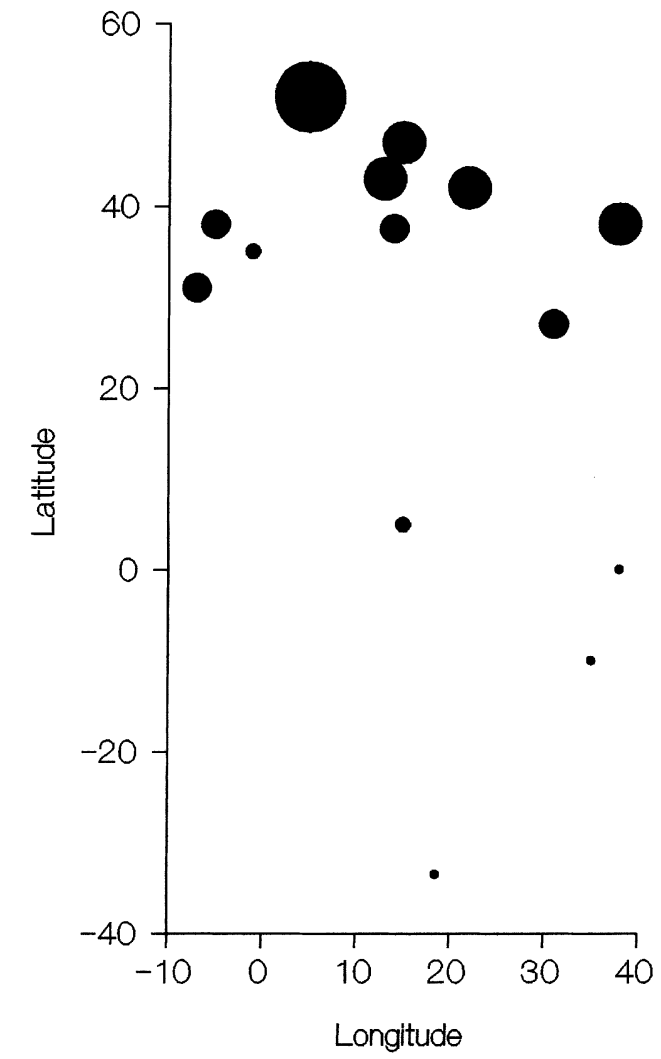

Fig. 4 Schematic map of multivariate morphological variation in the 14 subspecies of Apis mellifera analysed, using the first principal component of the phylogenetic $(\mathrm{P})$ values derived from PVR analysis of the 39 characters. The size of the circles indicates the relative values of multivariate scores, and large circles represent high scores (bees with a higher combined phylogenetic component).

Thus, departures from expected geographical variation under a simple north-south cline model observed in total values are a function of the specific (S) component, thus reflecting local adaptations in morphology. This is in accordance with the standard interpretations of partition methods (sensu Harvey \& Pagel, 1991), in which the P component expresses evolution by genetic drift or stabilizing selection, shared by all taxa in a clade, whereas variation in the $\mathrm{S}$ component reflects responses to directional selection independently in each lineage (Cheverud et al., 1985; Martins \& Hansen, 1996).

Size and shape variation in honey bees have usually been interpreted in terms of adaptations to local environmental conditions, and at large geographical scales they are expected to follow Bergmann's, Allen's and Glover's rules (Ruttner, 1988). In this analysis, body size variables and the first principal component of total values of the 39 characters (that can be interpreted as a multivariate expression of size variation), tend to have a large-scale geographical structure, with larger 
subspecies occurring in Europe and smaller subspecies in tropical Africa, as predicted by Bergmann's rule (Fig. 3). This geographical pattern is disturbed by some African subspecies, such as A.m. capensis. Although this subspecies disturbs linearity in the geographical variation patterns, it indeed supports an adaptive interpretation of morphological variation under Bergmann's rule. This occurs because A.m. capensis is restricted to an extreme position in southern Africa, and is a very peculiar local variation of $A$. mellifera, in terms of its biological and social characteristics (Ruttner, 1988). As these characteristics are local adaptations, they must be expressed only in the S component and, consequently, in the first phylogenetic principal component (PCA on $P$-values from PVR) these deviations in fact disappear (Fig. 4).

The characters of wing venation, on the other hand, show a very high phylogenetic correlation, indicating an elevated $\mathrm{P}$ component, but usually with lower spatial patterns. This can be interpreted by neutral (nonadaptive) evolution, in which the magnitude of divergence is proportional to time of divergence. This neutral model for the evolution of wing venation and nonallometric shape characters has already been discussed by Wagner (1990), who used these traits to construct a phylogeny to be used as a null hypothesis for studying evolution of morphological integration in Mediterranean races of A. mellifera. This neutral interpretation is strongly supported by the analyses presented here.

Colour variation usually does not show a significant correlation with either of the two referential dimensions. This lack of correlation could result from small-scale random variation in these traits, in both the phylogenetic and the spatial dimensions of variability. These small-scale variations could also be interpreted as resulting from measurement errors, which might be larger in these variables than are any spatial or phylogenetic effects on the data.

It is possible to conclude that phylogeographical autocorrelation analysis of morphological variation in honey bees can be a useful way to understand the evolutionary processes at the population level in these organisms. Also, it indicates how both adaptive and stochastic (neutral) evolution have affected distinct morphological traits. The most difficult problem in the application of this approach to other organisms is the absence of detailed phylogenies, especially at the population level, constructed using molecular techniques such as mtDNA. However, it is expected that this problem will be solved in the next few years, in view of the increasing number of such studies in many kinds of animals and plants. In this way, the combination between historical and geographical patterns of variation will become a very important approach in evolu- tionary and biogeographical studies at the populational level.

\section{Acknowledgements}

We thank L. M. Bini, E. P. Martins, M. Lynch, S. F. dos Reis and M. J. Cavalcanti for many discussions about linking comparative methods, multivariate generalization of PVR and population genetics. L. M. Bini and two anonymous referees provided many suggestions that improved the original manuscript. We would like to dedicate this paper to the memory of $F$. Ruttner, for his many contributions to bee biology and multivariate morphometrics. This work was supported by Conselho Nacional de Desenvolvimento Científico e Tecnológico (CNPq), FUNAPE-PRRPG/UFG and CAPES of Brazil, through many grants.

\section{References}

ARIAS, M.C. AND SHEPPARD, w. S. 1996. Molecular phylogenetics of honey bee subspecies (Apis mellifera L.) inferred from mitochondrial DNA sequence. Mol. Phyl. Evol., 5, 557-566.

AVISE, J. C., ARNOLD, J., BALL, R. M., BERMINGHAN, E., LAMB, T., NEIGEL, J. E. ET AL. 1987. Intraspecific phylogeography: the mitochondrial DNA bridge between population genetics and systematics. Ann. Rev. Ecol. Syst., 18, 489-522.

CHeVerud, J. M., DOW, M. M. AND LeUtenegGer, w. 1985. The quantitative assessment of phylogenetic constraints in comparative analyses: sexual dimorphism in body weight among primates. Evolution, 39, 1335-1351.

CORNUET, J. M. AND GARNERY, L. 1991. Mitochondrial DNA variability in honey bees and its phylogeographic implications. Apidologie, 22, 627-642.

CROZIER, R. H. 1990. From population genetics to phylogeny: uses and limits of mitochondrial DNA. Aust. Syst. Bot., 3, 111-124.

CUSHMAN, J. H., LAWTON, J. H. AND MANLY, B. F. J. 1993. Latitudinal patterns in European ant assemblages: variation in species richness and body size. Oecologia, 95, 30-37.

DINIZ-FILHO, J. A. F., SANT'ANA, C. E. R. AND BINI, L. M. 1998. An eigenvector method for estimating phylogenetic inertia. Evolution, 52, 1247-1262.

EDWARDS, S. V. AND KOT, M. 1995. Comparative methods at the species level: geographic variation in morphology and group size in grey-crowned babblers (Pomatostomus temporalis). Evolution, 49, 1134-1146.

FELSENSTEIN, J. 1985. Phylogenies and the comparative method. Am. Nat., 125, 1-15.

GitTleman, J. L. AND KOT, M. 1990. Adaptation: statistics and a null model for estimating phylogenetic effects. Syst. Zool., 39, 227-241.

HANSEN, T. F. AND MARTINS, E. P. 1996. Translating between microevolutionary process and macroevolutionary patterns: the correlation structure of interspecific data. Evolution, 50, 1404-1417. 
HARVEY, P. H. AND PAGEL, M. 1991. The Comparative Method in Evolutionary Biology. Oxford University Press, Oxford.

JACKSON, D. A. 1993. Stopping rules in principal component analysis: a comparison of heuristical and statistical approaches. Ecology, 74, 2204-2214.

JOHnSON, R. A. AND WICHERN, D. W. 1992. Applied Multivariate Statistical Analysis. Prentice Hall, London.

MARTINS, E. P. AND HANSEN, T. F. 1996. The statistical analysis of interspecific data: a review and evaluation of phylogenetic comparative methods. In: Martins, E. P. (ed.) Phylogenies and the Comparative Method in Animal Behaviour, pp. 22-75. Oxford University Press, Oxford.

ODEN, N. L. AND SOKAL, R. R. 1992. An investigation of threematrix permutation tests. J. Classif., 9, 275-290.

RODERICK, G. K. 1996. Geographic structure of insect populations: gene flow, phylogeography and their uses. Ann. Rev. Ecol. Syst., 41, 325-352.

ROHLF, F. J. 1989. NTSYS-PC: Numerical taxonomy and multivariate analysis system. Exeter Software, New York.

RUTTNER, F. 1988. Biogeography and Taxonomy of Honeybees. Springer-Verlag, New York.

SMOUSE, P. E., LONG, J. C. AND SOKAL, R. R. 1986. Multiple regression and correlation extensions of the Mantel test of matrix correspondence. Syst. Zool., 35, 627-632.

SOKAL, R. R. 1986. Spatial data analysis and historical processes. In: Diday, E. (ed.) Data Analysis and Informatics IV, pp. 29-43. North-Holland Science Publishers, Netherlands.
SOKAL, R. R. AND JACQUEZ, G. M. 1991. Testing inferences about microevolutionary processes by means of spatial autocorrelation analysis. Evolution, 45, 152-168.

SOKAL, R. R. AND ODEN, N. L. 1978a. Spatial autocorrelation in biology. 1. Methodology. Biol. J. Linn. Soc., 10, 199228.

SOKAL, R. R. AND ODEN, N. L. 1978b. Spatial autocorrelation in biology. 2. Some biological implications and four applications of evolutionary and ecological interest. Biol. J. Linn. Soc., 10, 229-249.

SOKAL, R. R. AND ROHLF, F. J. 1995. Biometry, 3rd edn. W. H. Freeman, New York.

TAYLOR, C. M. AND GOTELLI, N. J. 1994. The macroecology of Cyprinella: correlates of phylogeny, body size and geographical range. Am. Nat., 144, 549-569.

VAN VOORHIERs, w. A. 1996. Bergmann size clines: a simple explanation for their occurrence in ectotherms. Evolution, 50, 1259-1264.

WAGNER, G. P. 1990. A comparative study of morphological integration in Apis mellifera L. Z. Zool. Syst. Evolut-forsch., 28, 48-61.

WARTENBERG, D. 1985. Canonical trend surface analysis: a method for describing geographical patterns. Syst. Zool., 34, 259-279.

ZINK, R. M. 1996. Comparative phylogeography in North American birds. Evolution, 50, 308-317. 